\title{
Facile one pot microwave enhanced multistep synthesis of novel biologically important scaffold spiro[indole-pyridopyrimidines]
}

\author{
Anshu Dandia*, Kapil Arya, Sarita Khaturia, and Priyanka Yadav \\ Department of Chemistry, University of Rajasthan, Jaipur 302 004, India \\ E-mail: dranshudandia@eth.net
}

(received 07 Feb 05; accepted 06 Apr 05; published on the web 14 Apr 05)

\begin{abstract}
A rapid and efficient one pot method for the preparation of novel spiro [indole-pyrido[2,3d]pyrimidines] by the reaction of 'insitu' generated spiro [indole-dihydropyridine] and urea / $\mathrm{CS}_{2}$ using basic alumina as solid support/ or few drops of DMF as homogenizer under microwave irradiation is reported. Excellent yields (85-89\%) and higher purity are obtained in mw enhanced one pot synthesis as compared to conventional procedure which required multistep processes using organic solvents and tedious workups.
\end{abstract}

Keywords: Microwave irradiation, spiro[indole-pyridopyrimidines], spiro[indole-pyridines]

\section{Introduction}

The pyrido-pyrimidine core has been a useful functionality for the development of biologically interesting molecules. ${ }^{1}$ Indolines, incorporating either pyridine or pyrimidines have been extensively studied due to their occurrence in nature and of wide biological importance. ${ }^{2}$

In spite, of the immense biological activities of pyrido[2,3-d]pyrimidines and spiro [indolepyridines/pyrimidines] no reports have appeared describing the synthesis of spiro [indolepyrido[2,3-d]pyrimidines]. Microwave-assisted reactions which required short reaction times are becoming more popular for organic chemists ${ }^{3}$ and have recently been reviewed. ${ }^{4}$ More interest has been focused on dry media synthesis under MW irradiation and especially by carrying out experiments with supported reagents on mineral oxides, due to its eco friendly nature and it allows reactions in open vessels (thus avoiding risk of high pressure development) and synthesis on a preparative scale. ${ }^{5}$ Therefore, in a continuation of our studies on microwave assisted reactions ${ }^{6}$, we wish to report a new one pot preparation for novel spiro indole derivatives incorporating the pyrido[2,3-d]pyrimidine system under microwave irradiation using basic alumina as solid support in better yields and higher purities by reacting 'in situ' generated spiro [indole-dihydropyridines] 5 with urea or $\mathrm{CS}_{2}$. 
Although some pyrido[2,3-d]pyrimidines have been synthesized under microwave conditions but they involved other routes. ${ }^{7}$ Furthermore, different substituents could be incorporated at various positions by adopting these new methods. Therefore, it was considered worthwhile to synthesize spiro [indole-pyrido[2,3-d]pyrimidines] derivatives by these routes, to make them available for biological screening in the search for better medicinal agents.

\section{Results and Discussion}

In present investigation of a solvent free one pot synthesis of $\mathbf{6}$ and $\mathbf{7}$, the intermediates $\mathbf{4 a - c}$ were obtained in quantitative yields in 4-6 min by the reaction of indole-2,3-dione (1) and substituted acetophenones (2) without formation of the expected products formed conventionally using diethylamine as a catalyst ${ }^{8}$ (3a-c). The use of basic alumina as the mineral support therefore eliminates the necessity of an external base / strong acid for the synthesis of required intermediate 4 which was formed in reasonable purity (TLC) and could be used as such for next step without further purification.

The intermediate spiro [indole-dihydropyridines] $\mathbf{5}$ are also formed in reasonable purity (TLC) in quantitative yield under identical conditions by reaction with malononitrile and ammonium acetate in 5-6 min. A prior patent mentioned the conventional synthesis of 5 by other tedious routes with no synthetic details. ${ }^{9}$ Compounds $\mathbf{5}$ were used as such for conversion to $\mathbf{6}$ and 7 using basic alumina as solid support. (Scheme 1).

The reaction has also been performed under neat conditions (without solvent, support or catalyst), However, no reaction occurred under neat conditions in case of compounds $\mathbf{5}$ and $\mathbf{6}$, but could be made successful by adding a few drops of DMF. The role of DMF can be explained as an energy transfer agent and homogenizer to increase the reaction temperature. ${ }^{10,11}$ No formation of any detectable by-products were observed, which implies a drying effect. This method has the advantage of complete elimination of solvent for adsorption of reactant and desorption of product from the recyclable solid support. However, products are formed in comparatively lower yield and purity in this case as compared to the solid supported method.

For comparative studies some compounds were also synthesized under thermal conditions (Table 1). Conventional synthesis of $\mathbf{6}$ and $\mathbf{7}$ suffers from many disadvantages such as multistep reaction procedures, long reaction periods, low yields, and the use of strong acid / bases and hazardous solvents.

Hence, we have developed a new, economical, safe, environmentally benign one pot synthesis of novel spiro [indole-pyrido(2,3-d)pyrimidines] under microwave irradiation. The synthesis gives excellent yields of the required products 6 and 7 (85-89 \%) in 11-13 min. 


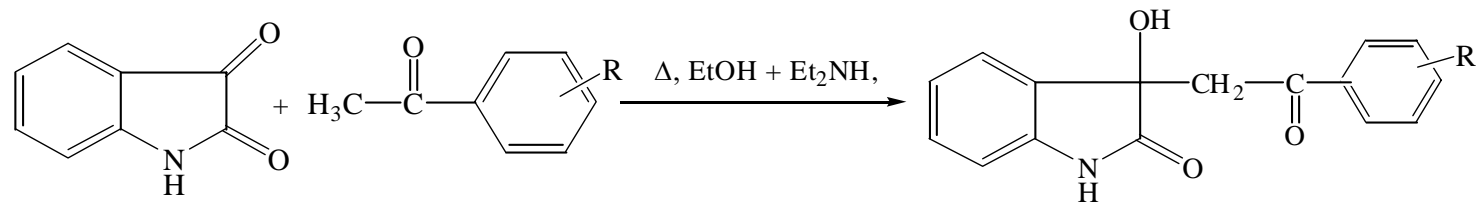

1

2a-c

2a : $\mathrm{R}=4-\mathrm{F}$

2c $: \mathrm{R}=4-\mathrm{OCH}_{3}$

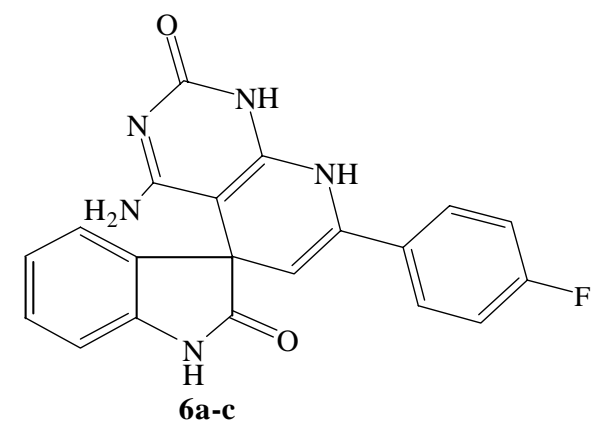

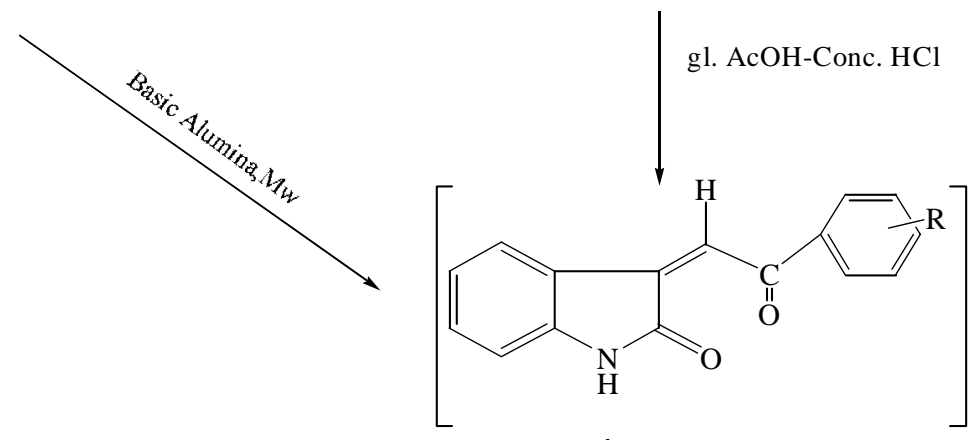

4a-c,

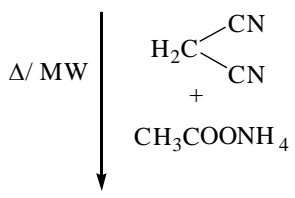

6a-c<smiles>[R]Cc1ccccc1C1=CC2(C(=O)Nc3ccccc32)c2c([nH]c(=S)[nH]c2=S)N1</smiles>
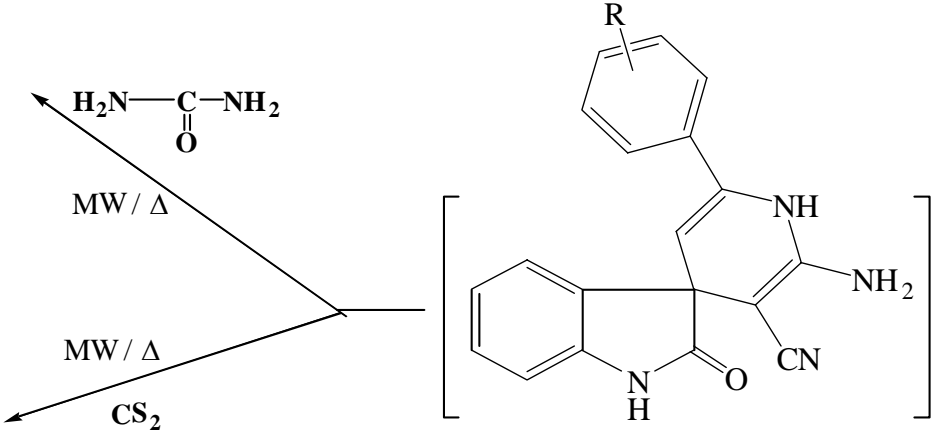

5a-c

7a-c

Scheme 1 
Table 1. Comparative study of the synthesis of 5a, 6a, 7a

\begin{tabular}{|c|c|c|c|c|c|}
\hline \multirow[t]{2}{*}{ Entry } & \multirow[t]{2}{*}{ Method } & \multirow[t]{2}{*}{$\operatorname{Temp}^{\mathrm{a}} \cdot\left({ }^{\circ} \mathrm{C}\right)$} & \multirow[t]{2}{*}{ Reaction time (min.) } & \multicolumn{2}{|c|}{ Isolated Yield (\%) } \\
\hline & & & & MW & $\Delta$ \\
\hline \multirow[t]{4}{*}{$5 a$} & $\Delta(\mathrm{EtOH})$ & Reflux & 540 & - & 70 \\
\hline & MW (Neat) & 82 & 10 & Nil & - \\
\hline & MW (Neat $+\varepsilon$ DMF) & 128 & 5 & 85 & - \\
\hline & MW (Basic alumina) & 130 & 4 & 96 & Traces $^{\mathrm{b}}$ \\
\hline \multirow[t]{4}{*}{$6 a$} & $\Delta(\mathrm{EtOH})$ & Reflux & 420 & - & 62 \\
\hline & MW (Neat) & 72 & 12 & Nil & - \\
\hline & MW (Neat $+\varepsilon$ DMF) & 125 & 6 & 80 & - \\
\hline & MW (Basic alumina) & 128 & 3 & 89 & Traces $^{b}$ \\
\hline \multirow[t]{4}{*}{$7 a$} & $\Delta$ ( pyridine) & Reflux & 850 & - & 60 \\
\hline & MW (Neat) & 98 & 10 & 60 & - \\
\hline & MW (Neat $+\varepsilon$ DMF) & 115 & 5 & 80 & - \\
\hline & MW (Basic alumina) & 135 & 2 & 85 & Traces $^{b}$ \\
\hline
\end{tabular}

${ }^{a}$ Final temperature is measured at the end of microwave irradiation by introducing a glass thermometer in the reaction mixture in the beaker.

${ }^{b}$ Yield under identical thermal condition of time, temperature and reaction vessels as under microwaves.

\section{Experimental Section}

General Procedures. Melting points were determined in open glass capillaries and were uncorrected. Thin layer chromatography on silica gel ' $G$ ' coated glass plates using benzene, ethanol $(8: 2)$ as eluent was used for monitoring the progress of the reactions. IR spectra (KBr) were recorded on a Magna FT IR-550 spectrophotometer, ${ }^{1} \mathrm{H}$ and ${ }^{13} \mathrm{C}$ NMR spectra $\left[\mathrm{CDCl}_{3}\right]$ were taken on a Bruker-300DX spectrometer at 300 and $200 \mathrm{MHz}$ respectively, using TMS as an internal standard for PMR and hexafluorobenzene as external standard for ${ }^{13} \mathrm{C}$ NMR. Mass spectra were recorded on Jeol D-300 spectrometer at an ionisation potential of 70 e.v. 
Microwave assisted reactions were carried out in a household MW oven (Panasonic-NN-781JF) equipped with inverter technology (generating fixed frequency through out the required time) for realistic control of the microwaves operating at $1000 \mathrm{~W}$ generating $2450 \mathrm{MHz}$ frequency.The apparatus was modified for laboratory applications, equipped with magnetic stirror and an external reflux condenser. All ketones were purchased from Aldrich Chemical Co. and were used as received.

\section{1,3-Dihydro-3-[2-(4-fluorophenyl)-2-oxoethylidene]indol-2(1H)-one(4a)}

Microwave mediated synthesis. Indole 2,3-dione (1) (1.47 g, $10 \mathrm{mmol})$ and 4fluoroacetophenone (2a) ( $1.38 \mathrm{~g}, 10 \mathrm{mmol}$ ) was adsorbed on basic alumina (20\% by weight of the reactants) via a solution in acetone, mixed thoroughly and irradiated under microwaves for 56 minutes at $128^{\circ} \mathrm{C}$. Since $100 \%$ conversion was observed with reasonable purity, (TLC) the reaction mixture was used as such for further reaction. For analytical and spectral studies it was recrystallized from methanol; (m.p. $186^{\circ} \mathrm{C}$, yield $=96 \%$ ).

Compounds $\mathbf{4 b , c}$ were prepared by using a similar procedure. The structure of the compounds was confirmed by IR and ${ }^{1} \mathrm{H}$ NMR and also by comparison with authentic samples prepared according to literature methods. ${ }^{12}$

\section{2'-Amino-3'-cyano-6'-(4-fluorophenyl)-1',4'-dihydro-spiro[3H-indole-3,4'(1-H)-pyridin]- 2(1H)-one 5 a}

Conventional Method. A mixture of 4a (2.67 g, $10 \mathrm{mmol})$, malononitrile (.66 g, $10 \mathrm{mmol})$, and ammonium acetate $(1.44 \mathrm{~g}, 20 \mathrm{mmol})$, in absolute ethanol $(50 \mathrm{~mL})$ was refluxed on water bath for 8-10 h, left at room temperature for $24 \mathrm{~h}$ and poured into crushed ice with constant stirring. The solid thus obtained was washed with water, dried and recrystallized from ethanol to give 5a. Microwave assisted synthesis. (a) Neat with few drops of DMF. An equimolar mixture of 4a (2.67 g, $10 \mathrm{mmol})$, and malanonitrile (.66 g, $10 \mathrm{mmol})$, and ammonium acetate $(.77 \mathrm{~g}, 10 \mathrm{mmol})$, with DMF( 109g, $1.5 \mathrm{mmol}$ ), was irradiated inside the microwave oven for an appropriate time (monitored by TLC), to give an oily product, which was solidified on standing, washed with water to give a crude product which was recrystallized from alcohol. (b) Using basic alumina as a solid support. A mixture of malononitrile ( .66 g,10 mmol ), and ammonium acetate (.77 g, $10 \mathrm{mmol}$ ) adsorbed on basic alumina (20\% by weight) with help of methanol was added to 4a (synthesized 'in situ') and irradiated inside the microwave oven at a power output of 100\% (1000 watts), for an appropriate time (Table 2). The product 5 was also found to be in reasonable purity (TLC) and hence used as such for the conversion into 6 and 7. For spectral and analytical studies, 5 was separated by filtration after eluting the reaction mixture with methanol. The excess solvent was evaporated on a roto-evaporator to give crystals of 5a, which were found to be pure (TLC). For analytical purpose compound was recrystallized from ethyl acetate .

The compounds 5b-c were prepared by following the same procedure and structure of compounds was confirmed by Spectral studies and literature m.ps. ${ }^{9,13}$ 
Table 2. Physical data of synthesized compounds 4a-c, 5a-c, 6a-c, 7a-c

\begin{tabular}{|c|c|c|c|c|}
\hline \multirow[t]{2}{*}{$\begin{array}{l}\text { Compound } \\
\text { No. }\end{array}$} & \multicolumn{2}{|c|}{$\begin{array}{c}\text { Reaction Time } \\
\text { (min.) / Yield (\%) }\end{array}$} & \multirow[t]{2}{*}{ Molecular formula } & \multirow[t]{2}{*}{ M. P. $\left({ }^{\circ} \mathrm{C}\right)$} \\
\hline & Classical method & Microwave method & & \\
\hline $4 a$ & $300 / 72$ & $5 / 98$ & $\mathrm{C}_{16} \mathrm{H}_{10} \mathrm{NO}_{2} \mathrm{~F}$ & $189-190^{8}$ \\
\hline $4 \mathrm{~b}$ & $300 / 68$ & $4 / 98$ & $\mathrm{C}_{17} \mathrm{H}_{13} \mathrm{NO}_{2}$ & $205-206^{12}$ \\
\hline 4c & $260 / 60$ & $4 / 98$ & $\mathrm{C}_{17} \mathrm{H}_{13} \mathrm{NO}_{3}$ & $239-240^{12}$ \\
\hline $5 a$ & $540 / 70$ & $4 / 96$ & $\mathrm{C}_{19} \mathrm{H}_{13} \mathrm{~N}_{4} \mathrm{FO}$ & $281-282^{13}$ \\
\hline $5 b$ & $480 / 72$ & $4 / 94$ & $\mathrm{C}_{20} \mathrm{H}_{16} \mathrm{~N}_{4} \mathrm{O}$ & $168-169^{9}$ \\
\hline $5 c$ & $520 / 65$ & $5 / 94$ & $\mathrm{C}_{20} \mathrm{H}_{16} \mathrm{~N}_{4} \mathrm{O}_{2}$ & $172-173^{9}$ \\
\hline $6 a$ & $420 / 62$ & 3/89 & $\mathrm{C}_{20} \mathrm{H}_{14} \mathrm{~N}_{5} \mathrm{FO}_{2}$ & $210-211$ \\
\hline $6 b$ & $400 / 60$ & $4 / 86$ & $\mathrm{C}_{21} \mathrm{H}_{17} \mathrm{~N}_{5} \mathrm{O}_{2}$ & $228-228$ \\
\hline $6 c$ & $410 / 60$ & $4 / 90$ & $\mathrm{C}_{21} \mathrm{H}_{17} \mathrm{~N}_{5} \mathrm{O}_{3}$ & $258-260$ \\
\hline $7 a$ & $840 / 60$ & $2 / 85$ & $\mathrm{C}_{20} \mathrm{H}_{13} \mathrm{~N}_{4} \mathrm{FOS}_{2}$ & $135-136$ \\
\hline $7 \mathrm{~b}$ & $920 / 62$ & $2 / 89$ & $\mathrm{C}_{21} \mathrm{H}_{16} \mathrm{~N}_{4} \mathrm{OS}_{2}$ & $177-178$ \\
\hline 7c & $840 / 60$ & $2 / 87$ & $\mathrm{C}_{21} \mathrm{H}_{16} \mathrm{~N}_{4} \mathrm{O}_{2} \mathrm{~S}_{2}$ & $205-206$ \\
\hline
\end{tabular}

\section{1,1',2,2'-Tetrahydro-4'-amino-7'-(4-fluorophenyl)-spiro[3H-indole-3,5'-(5H)pyrido(2,3-d) pyrimidine]-2,2'(1H)-diones (6a)}

Conventional method. A mixture of $5 \mathbf{a}(3.32 \mathrm{~g}, 10 \mathrm{mmol})$, and urea $(.9 \mathrm{~g}, 15 \mathrm{mmol})$, was heated on an oil bath at $120^{\circ} \mathrm{C}$ for $4 \mathrm{~h}$ with constant stirring. The temperature of reaction mixture was gradually raised to $180^{\circ} \mathrm{C}$ and it was heated at $220-30^{\circ} \mathrm{C}$ for $4 \mathrm{~h}$. After cooling the product was washed with water and finally with cold ethanol. The product thus obtained was dried and recrystallized from ethanol to give $\mathbf{6 a}$.

Microwave assisted synthesis. (a) Neat with few drops of DMF. An equimolar mixture of 5a, (3.32 g, $10 \mathrm{mmol}$ ) and urea (.6 g, $10 \mathrm{mmol}$ ), with DMF (.109 g, $1.5 \mathrm{mmol}$ ), was irradiated inside microwave oven for an appropriate time, (monitored by TLC), the solid obtained on cooling was washed with water and recrystallized from alcohol-water to give crude product. (b) Using basic alumina as solid support. To 5a (synthesized ' in situ') urea (.6 g, $10 \mathrm{mmol})$ separately adsorbed on basic alumina was added and irradiated inside the microwave oven at a power output of $100 \%$ (1000 watt), for the appropriate time (Table 2). After completion of the reaction (TLC) the recyclable inorganic solid support was separated by filtration after eluting the product with methanol to give white crystals of $\mathbf{6 a}$ which were found to be pure on TLC and do not require further recrystallization process.

The compounds $\mathbf{6 b}-\mathbf{c}$ were prepared by following the same procedure.

6a. Yield (89\%), m.p.210-211 ${ }^{\circ} \mathrm{C}$; IR (KBr)/ $\mathrm{cm}^{-}{ }^{1}$, 3420-3320 (NH \& NH ), 1720, 1690 (both $\mathrm{C}=\mathrm{O}), 1625(\mathrm{C}=\mathrm{N}), 740(\mathrm{C}-\mathrm{F}) ;{ }^{1} \mathrm{H}$ NMR $\left(\mathrm{CDCl}_{3}\right) \delta \mathrm{ppm}(\mathrm{s}, 1 \mathrm{H}, \mathrm{CH}), 5.78\left(\mathrm{br}, 2 \mathrm{H}, \mathrm{NH}_{2}\right), 6.92-$ 7.58 (m, 9H, Ar-H \& NH ), 8.57 (br, $1 \mathrm{H}, \mathrm{NH}), 11.02$ (br, $1 \mathrm{H}, \mathrm{NH}) ;{ }^{13} \mathrm{CNMR}\left(\mathrm{CDCl}_{3}\right) \delta: 84.2$

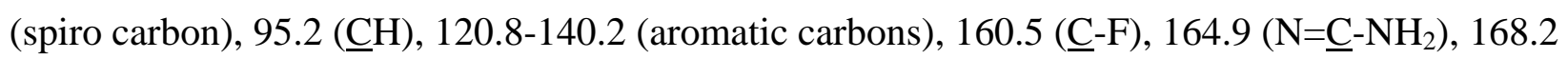




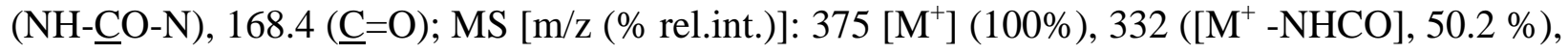
280 ([M $\left.\left.\mathrm{M}^{+}-\mathrm{C}_{6} \mathrm{H}_{4} \mathrm{~F}\right], 9.2\right), 194$ (13.2), 186 (31.1), 163 (28.5), 129 (63.7), 76 (50.4), 55 (19.2); Anal. Calc. For $\mathrm{C}_{20} \mathrm{H}_{14} \mathrm{~N}_{5} \mathrm{FO}_{2}(\mathrm{MW}=375)$, C: 64.00, H: 3.73, N: 18.66, Found C: 63.85, H: 3.82, $\mathrm{N}: 18.58 \%$.

6b. Yield (86\%), m.p 228-229 ${ }^{\circ} \mathrm{C}$; IR (KBr)/ $\mathrm{cm}^{-1}$, 3410-3320 (NH \& NH ), 1710, 1690 (both $\mathrm{C}=\mathrm{O}), 1620(\mathrm{C}=\mathrm{N}) ;{ }^{1} \mathrm{H}$ NMR $\left(\mathrm{CDCl}_{3}\right) \delta \mathrm{ppm} 2.15$ (s, 3H, $\left.\mathrm{CH}_{3}\right), 4.92(\mathrm{~s}, 1 \mathrm{H}, \mathrm{CH}), 5.92$ (br, 2H, $\mathrm{NH}_{2}$ ), 6.95-7.48 (m, 8H, Ar-H \& 1H, NH), 9.05 (br, IH, NH), 10.98 (br, IH, NH); ${ }^{13} \mathrm{CNMR}$ $\left(\mathrm{CDCl}_{3}\right) \delta: 22.8\left(\underline{\mathrm{CH}}_{3}\right), 77.4$ (spiro carbon), 94.6(CH), 119.6-145.2 (aromatic carbons), 165.3 $(\mathrm{NH}-\underline{\mathrm{CO}}-\mathrm{N}), 167.9\left(\mathrm{~N}=\underline{\mathrm{C}}-\mathrm{NH}_{2}\right), 169.6(\underline{\mathrm{C}}=\mathrm{O})$; Anal. Calc. For $\mathrm{C}_{21} \mathrm{H}_{17} \mathrm{~N}_{5} \mathrm{O}_{2}(\mathrm{MW}=371), \mathrm{C}$ : 67.92, H: 4.58, N: 18.86, Found C: 68.09, H: 4.46, N: 18.96\%.

6c. Yield (90\%), m.p. $258-260^{\circ} \mathrm{C}$; IR (KBr)/ cm- ${ }^{1}$, 3420-3330 (NH \& $\left.\mathrm{NH}_{2}\right), 1710,1680$ (both $\mathrm{C}=\mathrm{O}), 1615(\mathrm{C}=\mathrm{N}), 1120-1090(\mathrm{C}-\mathrm{O}-\mathrm{C}) ;{ }^{1} \mathrm{H}$ NMR $\left(\mathrm{CDCl}_{3}\right) \delta \mathrm{ppm} 3.78\left(\mathrm{~s}, 3 \mathrm{H}, \mathrm{OCH}_{3}\right) 5.02$ (s, 1H, CH), 6.02 (br, 2H, $\mathrm{NH}_{2}$ ), 6.75-7.45 (m, 8H, Ar-H \& 1H, NH) 9.05 (br, IH, NH), 11.05 (br, $\mathrm{IH}, \mathrm{NH}) ;{ }^{13} \mathrm{CNMR}\left(\mathrm{CDCl}_{3}\right) \delta: 57.9\left(\mathrm{OCH}_{3}\right), 77.4$ (spiro carbon), 93.7(타), 121.3-149.7 (aromatic carbons), 163.8 (NH-COO-N), $164.7\left(\mathrm{~N}=\underline{\mathrm{C}}-\mathrm{NH}_{2}\right), 169.5(\underline{\mathrm{C}}=\mathrm{O})$, Anal. Calc. For $\mathrm{C}_{21} \mathrm{H}_{17} \mathrm{~N}_{5} \mathrm{O}_{3}$ (MW=387), C: 65.11, H: 4.39, N: 18.08, Found C: 65.24, H: 4.48, N: 18.15\%.

\section{1,1',2,2',3',4'-Hexahydro-7'(4-fluorophenyl)-spiro[3H-indole-3,5'-(5H)pyrido(2,3-d) pyrimidine]-2',4'(1H, 3H)-dithione (7a)}

Conventional Method. A mixture of 5a (3.32 g, $10 \mathrm{mmol}$,) and carbon disulphide (2.5 mL, $40 \mathrm{mmol}$,) in pyridine (15 mL) was refluxed for $15 \mathrm{~h}$ After cooling, the excess pyridine was removed by distillation under reduced pressure and the residue was washed with water and cold ethanol. The crude product thus obtained was recrystallized from DMF-ethanol (1:10) to give 7a. Microwave assisted synthesis. (a) Neat with few drops of DMF. A mixture of 5a (3.32 g, $10 \mathrm{mmol}$,) and carbon disulphide (15mL) with DMF(.109 g, $1.5 \mathrm{mmol})$ was irradiated inside microwave oven for an appropriate time (monitored by TLC), to give crude product which was purified by recrystallization from ethanol. (b) Using basic alumina as solid support. A mixture of 5a (synthesized 'in situ') and carbon disulphide $(1.8 \mathrm{~mL}, 30 \mathrm{mmol}$ ) adsorbed on basic alumina was irradiated for appropriate time (Table 2). After completion of reaction (monitored by TLC), the product was obtained by extraction from methanol and recrysatllized from ethanol to give crystals of 7a.

The compound $\mathbf{7 b - c}$ was prepared by following the same procedure.

7a. Yield (85\%), m.p. 135-136 ${ }^{\circ}$; IR (KBr)/ cm- ${ }^{1}$, 3320-3280 (NH), 1720 (C=O), 1215-1190 $(\mathrm{C}=\mathrm{S}), 740(\mathrm{C}-\mathrm{F}) ;{ }^{1} \mathrm{H}$ NMR $\left(\mathrm{CDCl}_{3}\right) \delta \mathrm{ppm} 4.98$ (s, 1H, CH), 6.95-7.48 (m, 10H, Ar-H \& NH), 9.02 (br, 1H, NH), 11.12 (br, 1H, NH); ${ }^{13} \mathrm{CNMR}\left(\mathrm{CDCl}_{3}\right) \delta: 86.5$ (spiro carbon), 91.2 (C-C=S), 93.2(태), 121.8-138.5 (aromatic carbons), 158.3 ($-\mathrm{F}), 168.2(\underline{\mathrm{C}}=\mathrm{O}), 172.0(-\underline{\mathrm{C}}=\mathrm{S}), 192.0(-$ $\underline{\mathrm{C}}=\mathrm{S}$ ); Anal. Calc. For $\mathrm{C}_{20} \mathrm{H}_{13} \mathrm{~N}_{4} \mathrm{FOS}_{2}$ (MW=408), C: 58.82, H: 3.18, N: 13.72, Found C: 58.64, $\mathrm{H}: 3.05, \mathrm{~N}: 13.64 \%$.

7b. Yield (89\%), m.p.=177-178 ${ }^{\circ} \mathrm{C}$; IR (KBr)/ cm- ${ }^{1}$, 3310-3290 (NH), 1710 (C=O), 1210-1180 $(\mathrm{C}=\mathrm{S}) ;{ }^{1} \mathrm{H}$ NMR $\left(\mathrm{CDCl}_{3}\right) \delta \mathrm{ppm} 2.10$ (s, 3H, $\mathrm{CH}_{3}$ ), 4.85 (s, 1H, CH), 6.92-7.35 (m, 10H, Ar-H \& 
$\mathrm{NH}$ ), 9.05 (br, 1H, NH), 11.06 (br, 1H, NH); ${ }^{13} \mathrm{CNMR}\left(\mathrm{CDCl}_{3}\right) \delta: 21.8\left(\mathrm{C}-\underline{\mathrm{CH}}_{3}\right), 77.6$ (spiro carbon), $93.8(\underline{\mathrm{C}}-\mathrm{C}=\mathrm{S}), 94.5(\underline{\mathrm{CH}}), 119.2-145.6$ (aromatic carbons), 169.2( $\underline{\mathrm{C}}=\mathrm{O})$,177.5 (- $\underline{\mathrm{C}}=\mathrm{S})$, 191.8(- $\underline{\mathrm{C}}=\mathrm{S})$; Anal. Calc. For $\mathrm{C}_{21} \mathrm{H}_{16} \mathrm{~N}_{4} \mathrm{OS}_{2}$ (MW=404), C: 62.37, H: 3.96, N: 13.86, Found C: 62.49, H: 4.09, N: 13.97\%.

7c. Yield (87\%), m.p 205-206 ${ }^{\circ} \mathrm{C}$; IR (KBr)/ cm- ${ }^{1}$, 3320-3270 (NH), 1715 (C=O), 1215-1190 $(\mathrm{C}=\mathrm{S}), 1120-1090$ (C-O-C); ${ }^{1} \mathrm{H}$ NMR $\left(\mathrm{CDCl}_{3}\right) \delta \mathrm{ppm} 3.76$ (s, 3H, OCH $) 5.12$ (s, 1H, CH), 6.89$7.56(\mathrm{~m}, 8 \mathrm{H}, \mathrm{Ar}-\mathrm{H} \&$ \& $\mathrm{H}, \mathrm{NH}), 9.06$ (br, 1H, NH), 11.02 (br, 1H, NH); ${ }^{13} \mathrm{CNMR}\left(\mathrm{CDCl}_{3}\right)$ $\delta: 57.6\left(\mathrm{OCH}_{3}\right), 77.6$ (spiro carbon), 94.2 ( $\left.\underline{\mathrm{C}}-\mathrm{C}=\mathrm{S}\right), 95.6(\underline{\mathrm{CH}}), 118.0-146.8$ (aromatic carbons),167.5( $\underline{\mathrm{C}}=\mathrm{O}), 176.3(-\mathrm{C}=\mathrm{S}), 191.6(-\mathrm{C}=\mathrm{S})$; Anal. Calc. For $\mathrm{C}_{21} \mathrm{H}_{16} \mathrm{~N}_{4} \mathrm{O}_{2} \mathrm{~S}_{2}(\mathrm{MW}=420)$, C: 60.00, H: 3.80, N: 13.33, Found C: 60.09, H: 3.91, N: 13.25\%.

\section{Acknowledgements}

Financial assistance from C.S.I.R. (01(1907)/03/EMR-II), and U.G.C .New Delhi is gratefully acknowledged. We are also thankful to RSIC, IIT Delhi for the elemental and spectral analyses.

\section{References}

1. (a) Blankley, C. J.; Dherty, A. M.; Hamby, J. M.; Panik, R. L.; Schroeder, M. C.; Showalker, H. D. H.; Connolly, C. PCT Int. Appl. WO 9615, 1996, 128, US Appl, WO , 1994, 339,051; Chem. Abstr. 1995, 125, 114688s. (b) Piper, J. R.; Ramamurty, B.; Johnson, C. A.; Titer, G. M.; Sirotnak, F. M. J. Med. Chem. 1999, 39, 614. (c) Richard, C. P.; Y-Gui, Gu; Chin- Hung, L.; Erol, K.; Bayburt, J.; Mckie, K. M.; Alexander, K. L.; Wismer, C. T.; Mikusa, J.; Michael, F. J.; Elizabeth, A. K.; Shripad, S. B. J. Med. Chem. 2003, 46, 5249. (d) Bhagwat, S. S.; Perner, R. J.; Gu, Y. G., US 6,030,969, 2000; Chem. Abstr. 2000, 132,180592h. (e) Ogino, T.; Furukuwa, K. Eur. Pat. Appl. Pat. Appl. EP 994,113, 2000, JP Appl 1998/293, 250; Chem. Abstr. 2000, 132, 279228f. (f) Motto, H.; Yahio, P.; Isunco, I.; Onanj, R. J. M.; Ichiro, I.; Yoshia, M. Jpn Kokai TokkyoKoho JP 01,1989, 143, 895; Chem. Abstr. 1990, 112, 7859k. (g) Ganjee, A.; Adair, O.; Queener, S. F. Biorg \& Medicinal Chem. 2001, 9, 2929.

2. (a) Ona, K.; Sasajima, K.; Katsube, T. J.; Yamamoto, H. Japan Kokai 1978, 786, 8784; Chem. Abstr. 1978, 89, 129425j. (b) Smimoto Chemicals Co. Ltd. Belg. Pat. 1978, 897517; Chem. Abstr. 1979, 90, 121443p. (c) Cavalleto, C. J.; Gray, A. P. US Pat, 3409626, 1968; Chem. Abstr. 1979, 90, 47306q. (d) Hoechst, A. G., Japan Kokai 1978, 78135578; Chem. Abstr. 1979, 90, 137699n. (e) Ong, H. H.; Profit, J. A. US Pat. 1980, 4209625; Chem. Abstr. 1980, 93, 204474n. (f) Ong, H. H.; Agnew, M. N. J. Heterocycl. Chem. 1981, 18, 815. (g) Atlia, A.; Michael, M. Pharmazie 1982, 37, 551. (h) Matieson, D. S.; Bleebaum, M. S.; 
Bachtold, R. A.; Campbell, J. D.; Hillsek, R. J. J. Org. Chem. 1978, 34, 950. (i) Tokutake, N., Brit. Pat., 1468374, 1977; Chem. Abstr. 1977, 87, 102370j.

3. (a) Katritzky, A. R.; Cai, C.; Suzuki, K.; Singh, S. K. J. Org. Chem. 2004, 69, 811. (b) Katritzky, A. R.; Majumder, S. ARKIVOC 2003, (xiii), 74. (c) Katritzky, A. R.; Singh, S. K. ARKIVOC 2003, (viii), 68. (d) Krutosíková, A.; Lácová, M.; Dandárová, M.; Chovancová, J. ARKIVOC 2000, (i), 409. (e) Patel, V. M.; Desai, K. R. ARKIVOC 2004, (i), 123.

4. (a) Kappe, C. O. Angew. Chem. Int. Ed. 2004, 43, 6250. (b) Hoz, A. D.; Diaz-Ortiz, A.; Moreno, A. Current Organic Chemistry 2004, 8(10), 903. (c) Nüchter, M.; Ondruschka, B.; Bonrath, W.; Gum, A. Green Chemistry 2004, 6, 128. (d) Loupy, A. In Microwaves in Organic Synthesis; Wiley-VCH: Weinheim (Germany), 2002, ISBN 3-527-30514-9; (e) Lidstrom, P.; Tierney, J.; Wathey, B.; Westman, J. Tetrahedron 2001, 9225; (f) Varma, R. S. Green Chemistry 1999, 43, 1. (g) Loupy, A.; Perreux, L. Tetrahedron 2001, 57, 9199.

5. (a) Boruah, B.; Boruah, J.; Prajapati, D.; Sandhu, J. C.; Gosh, A. C. Tetrahedron Lett. 1996, 37, 4203. (b) Loupy, A.; Petit, A.; Hamelin, J.; Texier-Boullet, F.; Jacqualt, P.; Mathi, D. Synthesis 1998, 1213. (c) Tanaka, K. In solvent-free organic synthesis; Wiley-VCH: Weinheim (Germany), 2003; ISBN 3-527-30612-9.

6. (a) Dandia, A.; Sati, M.; Arya, K.; Sarawgi, P.; Loupy, A. ARKIVOC 2005, (i), 105. (b) Dandia, A.; Singh, R.; Sarawgi, P. J. Fluorine Chem. 2004, 125, 1835. (c) Dandia, A.; Arya, K.; Sati, M.; Gautum, S. Tetrahedron 2004, 60, 5253. (d) Dandia, A.; Singh, R.; Saha, M.; Shivpuri, A. Die Pharmazie. 2002, 57, 602; (e) Dandia, A.; Sachdeva, H.; Singh, R. Synth. Commun. 2001, 31, 1879. (f) Dandia, A.; Sati, M.; Loupy A. Green Chemistry 2002, 599.

7. Quiroga, J.; Cisneros, C.; Insuasty, B.; Abonia, R.; Nogueras, M.; Sanchez, A. Tetrahedron Lett. 2001, 42, 5625. (b) Mont, N.; Texido, J.; Borrel, J. I.; Kappe, C. O. Terahedron Lett. 2001, 44, 5385.

8. Brandmann, H. B. J. Heterocycl. Chem. 1973, 10, 383.

9. Wentland, P. M. US Pat 4,959,363, Appl. 370,926; Chem. Abstr. 1980, 93, 204474n. (b) Naito, T.; Miyata, O.; Ninomiya, I. Furusokan Kaguku Toronkai Koen Yoshishu 1979, 12, 126; Chem. Abstr. 1980, 93, 95470h.

10. Perez, R.; Perez, E.; Suarez, M.; Gonzalez, L.; Loupy, A.; Jimeno, M. L.; Ochoa, C. Org.Prep. Proced. Int. 1997, 29, 671.

11. Limousin, C. ; Cleophax, J.; Loupy, A. Petit, A. Tetrahedron 1998, 54, 13567.

12. Joshi, K. C.; Jain, R.; Garg, S. Pharmazie 1985, 40, 21.

13. Dandia, A.; Kumari, A.; Sehgal, V.; Rani, Indian. J. Chem. 1996, 35 B, 1314. 